\title{
Kinetic Energy-induced Growth Regimes of Nanocolumnar Ti Thin Films Deposited by Evaporation and Magnetron Sputtering
}

\author{
R. Alvarez ${ }^{1,2^{*}}$, A. Garcia-Valenzuela ${ }^{1}$, V. Rico ${ }^{1}$, J.M. Garcia-Martin ${ }^{3}$, J. Cotrino ${ }^{1,4}$, A.R. Gonzalez- \\ Elipe $^{1}$, A. Palmero ${ }^{1 *}$ \\ ${ }^{1}$ Instituto de Ciencia de Materiales de Sevilla, Avda. Américo Vespucio 49, 41092 Seville, Spain \\ ${ }^{2}$ Departamento de Física Aplicada I. Escuela Politécnica Superior, Universidad de Sevilla, c/ Virgen \\ de África 7, 41011 Seville, Spain \\ ${ }^{3}$ Instituto de Micro y Nanotecnología, IMN-CNM, CSIC (CEI UAM+CSIC), Isaac Newton 8, 28760 \\ Tres Cantos, Spain \\ ${ }^{4}$ Departamento de Física Atómica, Molecular y Nuclear. Universidad de Sevilla, Avda. Reina \\ Mercedes s/n., Seville, Spain \\ Abstract
}

We experimentally analyze different growth regimes of Ti thin films associated to the existence of kinetic energy-induced relaxation mechanisms in the material's network when operating at oblique geometries. For this purpose, we have deposited different films by evaporation and magnetron sputtering under similar geometrical arrangements and at low temperatures. With the help of a well-established growth model we have found three different growth regimes: i) low energy deposition, exemplified by the evaporation technique, carried out by species with typical energies in the thermal range, where the morphology and density of the film can be explained by solely considering surface shadowing processes, ii) magnetron sputtering under weak plasma conditions, where the film growth is mediated by surface shadowing mechanisms and kinetic-energy relaxation processes, and iii) magnetron sputtering under intense plasma conditions, where the film growth is highly influenced by the plasma, and whose morphology is defined by nanocolumns with similar tilt than evaporated films, but with much higher density. The existence of these three regimes explains the disparity of morphologies of nanocolumnar Ti thin films grown at oblique angles under similar conditions in the literature.

*E-mail: rafael.alvarez@icmse.csic.es, $\underline{\text { alberto.palmero@csic.es }}$ 


\section{Introduction}

The growth and characterization of nanocolumnar porous thin films have been the subject of intense research in the last years due to the wide range of applications based on them, e.g. in optics, biomedicine, sensors, microfluidics, LED fabrication, etc. [1-8]. These films have been classically deposited by the evaporation technique at glancing angles, which allowed the control of different microstructural features such as specific surface, density, pore distribution, pore connectivity, etc. [9-11]. Under this configuration, the deposition reactor is arranged to promote the arrival of sublimated species at a substrate along an oblique preferential direction, inducing surface shadowing processes and the formation of tilted nanocolumnar structures [12]. Remarkably, the implementation of this geometrical arrangement in magnetron sputtering reactors, the so-called magnetron sputtering at oblique angles (MS-OAD), has significantly widen the nanostructural possibilities of the technique, obtaining from dense and compact films to porous nanocolumnar arrays or even sponge-like structures, depending on the deposition conditions [13-18]. Yet, typical kinetic energies of deposition species in evaporation and magnetron sputtering are rather different, with values below $0.1 \mathrm{eV}$ in the former and up to few tens $\mathrm{eV}$ in the latter, which may have a profound influence on the film growth and the nanostructural development $[19,20]$. For instance, we showed that the low kinetic energy of evaporated atoms and their sustained proximity to the film surface at oblique geometries, previous to their deposition by the evaporation technique, made their movement in the gas phase deviate from straight trajectories, in a process dubbed Surface Trapping Mechanism. In reference [21] we demonstrated that the efficiency of this process ranged from 0 for metals to 1 for some oxides such as $\mathrm{SiO}_{2}$ or $\mathrm{Ta}_{2} \mathrm{O}_{5}$, and that it strongly affects the features and tilt angle of the nanocolumnar structures [22]. Conversely, when the kinetic energy of deposition species is above the binding energy of surface atoms (typically few eV), atomic mobility processes can be triggered at the landing point, preferentially in the direction of the lineal momentum, by means of so-called hyperthermal $(\mathrm{HT})$ relaxation mechanisms [23-26]. Interestingly, little is known on the role of HT processes at oblique geometries and their influence on the nanocolumnar growth and density of the films, which has motivated this work. For this, we have deposited Ti thin films by evaporation and MS-OAD and analyzed the results under the light of a well-tested growth model. We have chosen $\mathrm{Ti}$ as a reference material for several reasons: i) as a metal, the surface trapping processes can be neglected, which simplifies our study and the direct comparison between films, ii) its melting point is rather high, thus the role of thermally activated 
processes can be minimized when the growth takes place at room temperature, iii) there are numerous studies in the literature on MS-OAD of Ti which provide enough casuistic to validate our results in different deposition conditions and reactor geometries, and iv) there are some contradictory results in the literature regarding the growth of Ti thin films and the features of the nanocolumnar structures, which could be explained under the light of the results presented herein.

The existence of HT processes in the nanocolumnar growth of thin films by MS-OAD was experimentally proven in ref. [27]. In that publication, we deposited Ti thin films at oblique angles and at room temperature, finding slightly tilted columnar structures (their tilt angle with respect to the surface normal, $\beta$, was $\beta \sim 20^{\circ}$ for an incident angle of the deposition flux, $\alpha=80^{\circ}$ ). There, we deduced that HT processes were responsible for a so-called dragging mechanism, by which kinetic energy-induced atomic displacements in the film network compensate the natural tilt of the columnar structures towards the incoming flux of deposition species, making them more vertical. Based on a simple model that accounted for these processes (surface shadowing and HT processes), the growth of the film was simulated, finding nanostructures that accurately predicted the tilt angle of the nanocolumns and the film density under numerous deposition conditions. Remarkably, the solutions of the model in the absence of HT processes reproduced quite well the highly tilted columnar structures observed in evaporated Ti thin films under a similar geometrical arrangement, with $\beta \sim 50^{\circ}$. These results, however, contrast with others in the literature regarding the growth of Ti nanocolumns by MS-OAD, as it seems that similar geometrical configurations in the deposition reactor may lead to different nanocolumnar structures: in ref. [28], for instance, it was found that the tilt angle of Ti nanocolumns deposited by MS-OAD with $\alpha=80^{\circ}$ was $\beta \sim 50^{\circ}$, i.e. similar to the value obtained by evaporation, and quite far from the value reported in ref. [27] $\left(\beta \sim 20^{\circ}\right)$. Moreover, in ref. [29] it was found that the so-called Tangent Rule, an empirical equation that has been used to describe the relation between $\alpha$ and $\beta$ for evaporated films, was valid for MS-OAD of Ti thin films, which agrees with the results in ref. [28], suggesting that HT processes were not relevant in MS-OAD. This controversy was more evident in ref. [30], where nanocolumnar Ti thin films were grown by MS-OAD for biomedical applications in two different deposition reactors finding two different nanostructures: highly vertical nanocolumnar structures, resembling those in ref. [27], and highly tilted nanocolumnar structures, very similar to those reported in ref. [28], respectively, in an apparent contradiction that has not yet been explained. The main difference among these two later cases was the value of the electromagnetic power per unit area employed to maintain the plasma discharge. In order to clarify this issue, in this paper we analyze the influence 
of the electromagnetic power (for simplicity reasons, from now forth we will use the term power) on the growth of nanocolumnar Ti thin films and compare the results with those obtained by the evaporation technique and the predictions of the model developed in reference [27], both in the presence and in the absence of HT processes. Based on this analysis, the apparent controversy on the growth of Ti thin films by MS-OAD can be explained, and relevant conclusions can be drawn on the influence of kinetic energy-induced mechanisms on the growth of nanocolumnar Ti thin films at oblique geometries.

\section{Experimental Setup}

$\mathrm{Ti}$ thin films were grown in a vacuum reactor using the magnetron sputtering technique in the deposition setup shown in figure 1a. A $7.5 \mathrm{~cm}$ diameter titanium target was employed, placing the substrate holder at a distance of $7 \mathrm{~cm}$. A collimator made of aluminum with a $2 \mathrm{~cm}$ base length and $20^{\circ}$ angular aperture, already described in ref. [31], was employed to cover the $1 \mathrm{~cm} \times 1 \mathrm{~cm}$ substrates. The substrate holder was grounded and tilted $95^{\circ}$ with respect to the target surface to achieve an average incidence angle of deposition species at the substrate of $\alpha=80^{\circ}$ [29]. As shown in ref. [31], the collimator inhibits the deposition of a large number of low energy atoms that may arrive at the film surface from random directions, and only allows the deposition of relatively high energy ballistic species steaming from a very narrow part of the racetrack, which effectively acts as a nearly punctual source of deposition species. In figure $1 \mathrm{~b}$ we show a labeled photograph of our system. The electromagnetic power generator was set in DC mode, with the power ranging from $25 \mathrm{~W}$ to $400 \mathrm{~W}$. The base pressure of the deposition reactor was $7 \times 10^{-4} \mathrm{~Pa}$. Argon was employed as sputter gas, with a pressure of $p_{g}=0.15 \mathrm{~Pa}$. Data on the current and target potential appears in Table I, where it is shown that the target voltage changed very weakly with power, from $289 \mathrm{~V}$ to $316 \mathrm{~V}$, while the current varied from $0.09 \mathrm{~A}$ to $1.26 \mathrm{~A}$. Deposition time in each case was set to obtain films with thicknesses around $300 \mathrm{~nm}$. Substrate temperature was measured in the inner side of the collimator in all the cases (c.f. Table I). Yet, in one case, the substrate was intentionally heated up to $150{ }^{\circ} \mathrm{C}$ to check the influence of substrate temperature on the columnar morphology. Ion flux towards the film was measured using an energy-resolved mass spectrometer (EQP 500, Hiden Analytical Ltd), that measures the mass and energy of plasma ions. The orifice of the spectrometer $(0.05 \mathrm{~mm}$ of diameter) was positioned at a distance of $70 \mathrm{~mm}$ from the center of the target, at the same location where the substrates were placed during the deposition. Moreover, 
for comparison purposes, a Ti thin film was deposited by electron beam-assisted physical vapor deposition (i.e. by the evaporation technique) at a zenithal deposition angle of 80 o using the experimental set-up described in ref. [21]. Field Emission Scanning Electron Microscopy (FESEM)

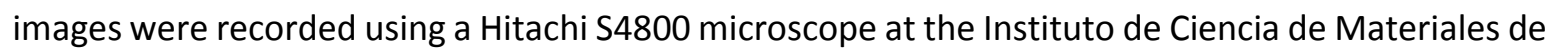
Sevilla (CSIC-US, Seville, Spain) as regular scanned images and as snapshots to avoid any signal drift during measurement. Rutherford Backscattering Spectroscopy (RBS) was employed to assess the number of atoms per unit area of the films. Experiments were carried out in the $3 \mathrm{MeV}$ tandem accelerator of the National Center for Accelerators (Seville, Spain) with a beam of $1.5 \mathrm{MeV}$ alpha particles and a passivated implanted planar silicon (PIPS) detector located at 165ㅇ scattering angle, with accumulated doses about $1.5 \mu \mathrm{C}$, and $\sim 1 \mathrm{~mm}$ beam spot diameter. The RBS spectra were simulated with the SIMNRA code [32], and the film density was calculated by dividing the number of atoms per unit area by the film thickness, as in ref. [27]. Moreover, to avoid any miscalculation on the film thickness, it was specifically measured at a position next to the spot of the RBS measurement on the samples' surface.

\section{Results and Discussion}

In figure 2 we show cross-sectional images of few selected Ti thin films grown for increasing values of power. As expected, films depict well-defined tilted nanocolumnar structures with $\beta$ ranging from $34^{\circ}$ to $50^{\circ}$, demonstrating a direct dependence between $\beta$ and power. This relation has been depicted in figure 3a for all the cases gathered in Table I (the dashed curve in figure 3a is included to guide the eyes). Moreover, in figure $3 \mathrm{~b}$ we show the values of the relative density of these films as a function of power, finding again a transition from $\sim 30 \%$ to $\sim 45 \%$ when the power varies from 25 to $400 \mathrm{~W}$. To further understand this dependence we have deposited a Ti thin film by evaporation under similar geometrical conditions, i.e. with $\alpha=80^{\circ}$, also displayed in figure 2 , where we determine the value $\beta=52^{\circ}$, included in figure 3a. Remarkably, and despite the fundamental differences between techniques, the tilt angle of the nanocolumns is rather similar in evaporation and in MS-OAD at high powers in figure 3a. Yet, it is noteworthy that column diameter seems to be much larger in evaporation (note the different magnification scales in the cross section images reported in figure 2), suggesting that the governing processes in either situation must be very different. This becomes evident in figure $3 b$, where we have also included the relative density 
of the evaporated film, which differs by factor around 2 with respect to the MS-OAD films grown at higher powers $(\sim 45 \%$ and $\sim 21 \%)$.

To shed some light on the growth mechanisms involved under each experimental condition, we have solved the model in ref. [27] in two different scenarios: i) when HT relaxation mechanisms are included, and ii) when they are excluded. The results of these simulations are depicted in figure 4, where we can clearly notice the appearance of columnar structures with $\beta=30^{\circ}$ when HT processes are taken into account. This nanostructure resembles that in ref. [27] and agrees with the reported value of $\beta$ at the lowest power (results of the model are depicted as straight solid lines in figure 3a). Moreover, in the absence of HT processes, the tilt angle of the structures, $\beta=49$, matches relatively well that obtained by MS-OAD at high powers, as well as by evaporation. Incidentally, the value of the density predicted by the model in the absence of HT processes matches quite well with that of the film grown by evaporation (see figure $3 \mathrm{~b}$ ), while it clearly fails to describe that of the high power cases. This good agreement between the model in the absence of HT processes and the features of the evaporated $\mathrm{Ti}$ film demonstrate that surface shadowing mechanisms are enough to explain main morphological features (column tilt angle and film density) of this film. To our knowledge, this is the first time in the literature that this conclusion has been quantitatively demonstrated. Moreover, the results of the model when the HT processes are included reproduce fairly well the tilt angle (figure 3a) and density (figure $3 b$ ) of the film grown by MS-OAD at the lowest power, pointing towards the dominance of HT and surface shadowing mechanisms during growth under these conditions. Remarkably, for increasing values of power, the nanostructural features of the films are not well calculated by the model, neither with nor without HT processes, demonstrating the existence of profound changes in the growth mechanisms. This can be clearly appreciated in figure 5, where we have plotted the value of $\beta$ as a function of the relative density of the films in either case and where we have included the three different growth regimes discussed above: i) low energy deposition, exemplified by evaporation, where surface shadowing governs the film growth, ii) MS-OAD under a weak plasma influence (low power), where HT relaxation processes compete with surface shadowing to generate the columnar microstructure, and iii) MS-OAD under an intense plasma influence (high power), where surface shadowing and HT processes are not enough to explain all nanostructural features of the films.

The atomistic processes governing the nanostructuration process by MS-OAD at high powers are quite puzzling. Regarding the results above, it is unlikely that HT processes become inhibited when 
solely increasing the power: according to the Thompson formula the energy distribution of sputtered species from the target mainly depends on the binding energy of target atoms and, thus, the energy distribution of sputtered species should be almost independent of power [19]. Yet, the difference in masses between $\mathrm{Ar}$ ions and $\mathrm{Ti}$ atoms may produce certain deviations from the Thompson formula, and a dependence of the energy of sputtered species on the sputter ion energy (i.e. with the target potential) is possible [19]. Nevertheless, and according to Table I, higher power values mainly produced an increase of the plasma current in our conditions, being the target potential (sputter ion energy) rather constant in all the cases. That is why, in the conditions studied in this paper, we rule out any relevant influence of power on the energy distribution of sputtered species and on the existence of HT processes.

Another additional mechanism that might be responsible for the reported transition from low to high powers can be associated to the existence of thermally-induced mobility processes, due to higher deposition rates and the progressive heating of the plasma gas for increasing powers [20]. However, all the films were grown at relatively low temperatures (c.f. Table I), defining an homologous temperature (film temperature divided by the melting temperature of the material) below 0.2 in all the cases, which, according to the Thornton Structure Zone Model, points to a negligible role of thermally induced relaxation processes $[33,34]$. To experimentally check this conclusion we have deposited a film under same conditions as before, using a power of $100 \mathrm{~W}$, but imposing a substrate temperature of $150 \stackrel{\circ}{ } \mathrm{C}$. The cross-sectional view of this film appears in figure 6 , where we can notice the existence of tilted nanocolumnar structures with $\beta=38^{\circ}$ and a density of $35 \%$ (values included in figure $3 a-b$ and 5 ), very similar to those found when the growth was carried out at low temperature. This means that an increase of temperature up to $150 \stackrel{\circ}{C}$ affects very little the film morphology. Moreover, depositions of Ti under similar geometrical conditions at higher substrate temperatures, up to $\sim 500{ }^{\circ} C$, were carried out in ref. [28], where it was obtained that thermally activated processes induce a more vertical columnar growth, i.e. a behavior opposite to what we find in figure $3 a$ when increasing the power. Consequently, and based on these evidences, we rule out any relevant influence of thermally activated processes in the reported transition with power in figures 3a-b.

The increase of deposition rate with power may also be responsible for the reported structural changes: usually it is relevant whenever the typical arrival time is in the same order of magnitude of the typical relaxation time of atoms on the film surface per unit time and area. In figure 7a we show 
the Ti deposition rate as obtained by RBS as a function of power, where we find a rather linear dependence. Even though thermally activated relaxation mechanisms have been shown to be negligible in our conditions, we cannot disregard the existence of HT relaxation processes on the film surface. Therefore, we cannot rule out that an increase of deposition rate with power can be responsible for the reported changes in figures 3a-b. Moreover, an additional effect that might explain the transition with power relies on the existence of higher energy fluxes in the plasma towards the film [23], e.g. ion current, flux of excited species in metastable states, flux of reflected high energy neutrals, etc., which might interact with the film surface during growth. To illustrate this behavior, in figure $7 \mathrm{~b}$ we show the flux of $\mathrm{Ar}^{+}$ions towards the film as obtained by the mass spectrometer as a function of power, which increases almost linearly, likewise the deposition rate (figure 7a). These values agree with ref. [35], where the energy fluxes towards the film were measured and where it was demonstrated that, in the case of $\mathrm{Ti}$, the dominant flux was associated to the radiation emitted from the target. These energy fluxes may indeed affect the atomistic processes during growth differently, bur their analysis in detail and their influence on the atomistic aggregation process are beyond the scope of this paper. That is why, and until further explication is found on the atomistic processes responsible for triggering such transition with power, we have decided to label each regime according to the existence of a weak or an intense plasma, instead of using any other power-related quantity such as the deposition rate or the ion current for instance.

In ref. [36] Shaginyan et al. proposed that under certain energetic plasma/film interaction conditions, the first monolayers of the growing film form a metastable hot thin solid layer that alters the way atoms incorporate into the film, from classic gas $\rightarrow$ solid mechanism to gas $\rightarrow$ liquid $\rightarrow$ solid paths, in a phenomenon that cannot be ruled out under the studied conditions and that could change the nature of the HT relaxation mechanisms. In any case, it is remarkable that the growth under intense plasma conditions produces nanocolumnar structures as tilted as those produced by evaporation. This explains why models that only consider the shadowing mechanism have been able to accurately predict the tilt angle of the nanocolumnar structures grown by MS-OAD or why the Tangent Rule [21], a typical relation employed in evaporation relating $\alpha$ and $\beta$, is valid in MS-OAD in some conditions. However, as we have demonstrated in figure 5 , this agreement is limited to the tilt angle of the nanocolumns, whereas the accurate description of any other morphological feature, such as the film density, requires the introduction of kinetic energy-induced or plasma-induced processes. These results also explain the diversity of the experimental data in the literature regarding the growth of Ti thin films: in figure 8 we show the different tilt angle of the structures for 
Ti grown by MS-OAD at incidence angles of about $80^{\circ}$, taken from different works in the literature, along with the results of the model solved for each experimental condition, with and without HT processes. In all the cases, we can check that either the experimental data corresponds to a weak plasma regime (ref. [27] and System 1 in ref. [30]), or they match quite well the tilt angle of the nanocolumns in the absence of HT processes (ref. [28] and System 2 in ref. [30]). The actual value of the density of the films is not known in this latter case, and they could correspond to films grown by low energy deposition species or, most likely, by MS-OAD under intense plasma conditions, thus clarifying the apparent controversy in the literature concerning the growth of nanocolumnar Ti thin films by MS-OAD. Moreover, considering the similar value of the target-to-substrate distance and power density, it is also remarkable the similarity between the results of Dervaux et al. in ref. [28], and our results under an intense plasma condition in figure $3 a$.

To our knowledge, it is the first time that these three growth regimes have been reported, linked to kinetic-energy induced relaxation mechanisms and the existence of a weak or an intense plasma. These two situations, even if the geometrical arrangement in the deposition reactors is the same, lead to rather different film morphologies, implying that film properties might also be quite different, e.g. hardness, elastic modulus, etc. [37-39]. While two of these regimes have been characterized in terms of atomistic processes, we have demonstrated that the third one is associated to the presence of an intense plasma. In our setup we could trigger the transition from a weak to an intense plasma growth regime by increasing the power from $25 \mathrm{~W}$ to $400 \mathrm{~W}$, although in other reactors, it is likely that different power coupling and specific plasma features might induce the transition at different values of the electromagnetic power. Moreover, we have also demonstrated quantitatively the intrinsic differences between evaporation and magnetron sputtering in terms of atomistic processes and plasma effects at room temperature that, to our knowledge, were not previously addressed in the literature. As a final remark, it is noteworthy that we use the oblique angle configuration because of the strong link existing between atomistic processes and the features of the nanocolumnar structures, the latter enhanced thanks to the use of a particle collimator. The results obtained herein should be in principle applicable to other geometries and configurations as well as to other materials. However, at classical non-oblique geometries the morphological changes would be less evident due to the compact structure of the films. All these issues could only be fully clarified once the relevant plasma-related mechanism could be identified, as well as the atomistic processes governing the film nanostructuration under intense plasma conditions, which will be studied in future works. 


\section{Conclusions}

In this work, we have experimentally analyzed the influence of kinetic energy-induced relaxation mechanisms in the network during the growth of Ti thin films when operating at oblique geometries. For this purpose, we have deposited different thin films by evaporation and magnetron sputtering under similar geometrical arrangements at low temperatures, and analyzed the features of the columnar morphology and the film density. With the help of a well-established growth model we have found three different growth regimes, each defined by a typical morphology and film density: i) low energy deposition, exemplified by the evaporation technique, carried out by species with typical energies much below the typical threshold to trigger atomic mobility in the film network, and where the morphology and density of the film can be explained by solely considering surface shadowing processes, ii) magnetron sputtering under weak plasma conditions, where the film growth is mediated by surface shadowing mechanisms and kinetic-energy induced relaxation processes in the film network, and iii) magnetron sputtering under intense plasma conditions, where the film growth is highly influenced by the plasma, and whose morphology is defined by nanocolumns with similar tilt as evaporated films, but with much higher density. The existence of these three regimes explains the disparity of morphologies of nanocolumnar Ti thin films grown at oblique angles under similar conditions that have been reported in the literature, and that we have tested by solving an atomistic growth model. Our results provide a framework to understand the influence of kinetic energy-induced relaxation mechanisms and the plasma on the development of the film nanostructure.

\section{Acknowledgements}

The authors thank the European Regional Development Funds program (EU-FEDER) and the MINECO-AEI (201560E055, MAT2014-59772-C2-1-P, and MAT2016-79866-R and network MAT2015-69035-REDC) for financial support. The authors also acknowledge the financial support of the University of Seville (V and VI PPIT-US). The National Center for Accelerators (Seville, Spain) is also acknowledged. 


\section{References}

[1] M. Martín, P. Salazar, R. Alvarez, A. Palmero, C. López-Santos, J.L. González-Mora, Agustín R. González-Elipe, Sensors and Actuators B 240, 37 (2017)

[2] M. Oliva-Ramírez, L. González-García, J. Parra-Barranco, F. Yubero, A. Barranco, A. R. GonzálezElipe, ACS Appl. Mater. Interfaces 5, 6743 (2013)

[3] J. Ollitrault, N. Martin, J. Y. Rauch, J. B. Sanchez, F. Berger, Mater. Lett. 155, 1 (2015).

[4] C. Sengstock, M. Lopian, Y. Motemani, A. Borgmann, C. Khare, P. J. S. Buenconsejo, T. A.

Schildhauer, A. Ludwig, M. Koller, Nanotechnology 25, 195101 (2014)

[5] Y. J. Yoo, J. H. Lim, G. J. Lee, K. I. Jang, Y. M. Song, Nanoscale 9, 2986 (2017)

[6] L. Yang, Y. Zhao, Y. Feng, J. S. Shan, X. Y. Liang, J. Huang, J. H. Mi, L. J. Wang, W. M. Shi, Appl. Surf. Sci. 363, 252 (2016).

[7] D. B. Polat, O. Keles, Thin Solid Films 589, 543 (2015)

[8] Y.J. Lee, Z.P. Yang, F.Y. Lo, J.J. Siao, Z.H. Xie, Y.L. Chuang, T.Y. Lin, J.K. Sheu, APL Materials 2, 056101 (2014)

[9] V Godinho, P Moskovkin, R Alvarez, J Caballero-Hernández, R Schierholz, B Bera, J Demarche, A Palmero, A Fernández and S Lucas, Nanotechnology 25, 355705 (2014)

[10] S. Liedtke, C. Gruener, J. W. Gerlac, B. Rauschenbach, Beilstein J. Nanotechnol. 9, 954 (2018)

[11] K. Kim, J. H. Park, H. Kim, J. K. Kim, E. F. Schubert, J. Cho, Appl. Phys. Letters108, 011910 (2016)

[12] A. Barranco, A. Borras, A.R. Gonzalez-Elipe, A. Palmero, Progress in Materials Science 76, 59 (2016)

[13] J.M. Garcia-Martin, R. Alvarez, A. Cebollada, A. Palmero, Appl. Phys. Lett. 97, 173103 (2010)

[14] D. Toledano, R. E. Galindo, M. Yuste, J.M. Albella, O Sanchez J. Phys. D: Appl. Phys. 46, 045306 (2013)

[15] C.M. Zhou, D. Gall, Appl. Phys. Lett. 88, 203117 (2006) 
[16] S.V. Kesapragada, D. Gall D Appl. Phys. Lett. 89203121 (2006)

[17] F. Ruffino and M. G. Grimaldi Nanosci. Nanotechnol. Lett. 4309 (2012)

[18] C. Patzig, T. Karabacak, B. Fuhrmann, B. Rauschenbach J. Appl. Phys. 104, 094318 (2008)

[19] Reactive Sputter Deposition. (Eds: D. Depla, S. Mahieu), Springer Series in Materials Science, Springer-Verlag, Berlin, Heildelberg 2008, ISBN 978-3-540-76662-9.

[20] A. Palmero, H. Rudolph, F.H.P.M. Habraken, Appl. Phys. Lett. 89, 211501 (2006)

[21] R. Alvarez, C. Lopez-Santos, J. Parra-Barranco, V. Rico, Angel Barranco, J. Cotrino, A. R. Gonzalez-Elipe, A. Palmero, J. Vac. Sci. Technol. B 32(4), 041802 (2014)

[22] C. Lopez-Santos, R. Alvarez, A. Garcia-Valenzuela, V. Rico, M. Loeffler, A.R. Gonzalez-Elipe, A. Palmero, Nanotechnology 27, 395702 (2016)

[23] G Abadias, W P Leroy, S Mahieu, D Depla, J. Phys. D: Appl. Phys. 46, 055301 (2013)

[24] X.W. Zhou and H.N.G. Wadley Surf. Sci. 43142 (1999)

[25] X.W. Zhou and H.N.G. Wadley, Surf. Sci. 431, 58 (1999)

[26] J.C.S. Kools J. Vac. Sci. Technol. A 23, 85 (2005)

[27] R Alvarez, J M Garcia-Martin, A Garcia-Valenzuela, M Macias-Montero, F J Ferrer, J Santiso, V Rico, J Cotrino, A R Gonzalez-Elipe, A Palmero, J. Phys. D: Appl. Phys. 49, 045303 (2016)

[28] J. Dervaux, P.-A Cormier, P. Moskovkin, O. Douheret, S. Konstantinidis, R. Lazzaroni, S. Lucas, R. Snyders, Thin Solid Films 636, 644 (2017)

[29] A. Siad, A. Besnard, C. Nouveau, P. Jacquet, Vacuum 131, 305 (2016)

[30] D. Medina-Cruz, M. U. Gonzalez, W. Tien-Street, M. Fernandez-Castro, A. Vernet-Crua, I. Fernandez-Martinez, L. Martinez, Y. Huttel, T. J. Webster, J.M. Garcia-Martin, Nanomedicine: Nanotechnology, Biology, and Medicine 17, 36 (2019)

[31] A. Garcia-Valenzuela, R. Alvarez, V. Rico, J. Cotrino, A.R. Gonzalez-Elipe, A. Palmero, Surf. Coat. Technol. 343, 172 (2018) 
[32] M. Mayer, SIMNRA User's Guide, Tech. Rep. IPP 9/113, Max-Plank Institut fur Plasmaphysik, Garching, Germany 1997.

[33] J.A. Thornton, J. Vac. Sci. Technol. 11666 (1974)

[34] J.A. Thornton J. Vac. Sci. Technol. 12830 (1975)

[35] S. Gauter, F. Haase, H. Kersten, Thin Solid Films 669, 8 (2019)

[36] L. R. Shaginyan, Y.J.Kim, J. G. Han, N.V. Britun, J. Musil, I.V. Belousov, Surf. Coat. Technol 202, 486 (2007)

[37] R. El Beainou, A. Chargui, P. Pedrosa, A. Mosset, S. Euphrasie, P. Vairac, N. Martin, Appl. Surf. Sci. 475, 606 (2019)

[38] R. El Beainou, R. Salut, L. Robert, J.-M. Cote, V. Potin, N. Martin, Materials Letters 232, 126 (2019)

[39] E Coffy, G. Dodane, S. Euphrasie, A. Mosset, P. Vairac, N. Martin, H. Baida, J.-M. Rampnoux, S. Dilhaire, J. Phys. D: Appl. Phys 50(48), 484005 (2017) 


\section{Figure Caption}

Figure 1.- a) Schematics of the experimental setup. b) Labeled photograph of the experimental setup.

Figure 2.- Cross-sectional images of Ti films grown at $25 \mathrm{~W}, 100 \mathrm{~W}, 200 \mathrm{~W}, 300 \mathrm{~W}$ and $400 \mathrm{~W}$ along with that obtained by evaporation. The average tilt angle of the columnar structures is also presented, with an error of $\pm 2^{\circ}$. Note the different scale for the evaporated film.

Figure 3.- Values of a) the tilt angle of the nanocolumnar structures, and b) the relative density of the films, represented as a function of power (dashed line is included to guide the eyes). The corresponding values for the evaporated film are depicted on the right. Results of the model, when hyperthermal processes are considered or inhibited, are also included as horizontal solid lines.

Figure 4.- Results of the model introduced in ref. [27], solved for the conditions of this paper, when hyperthermal processes are considered (left) and inhibited (right). The value of the tilt angle of the resulting nanostructures is also shown.

Figure 5.- Tilt angle of the nanocolumnar structures as a function of film density.

Figure 6.- Cross-sectional view of a film grown by magnetron sputtering at oblique angles using a power of $100 \mathrm{~W}$, heating up the substrate to $150{ }^{\circ} \mathrm{C}$ (see Table I).

Figure 7.- a) Deposition rate and b) $\mathrm{Ar}^{+}$flux towards the film in the conditions described in Table I.

Figure 8.- Tilt angle of nanocolumnar Ti thin films reported in the literature. We also include the values obtained by the model developed in ref. [27], modified for their respective geometrical conditions, solved when hyperthermal processes are considered and inhibited. 


\section{Table Caption}

Table I.- Value of the electromagnetic power, target potential, current, temperature and other data used to grow the films.

\begin{tabular}{|c|c|c|c|c|}
\hline $\begin{array}{l}\text { Power } \\
\text { (W) }\end{array}$ & $\begin{array}{l}\text { Target Potential } \\
\text { (V) }\end{array}$ & $\begin{array}{l}\text { Current } \\
\text { (A) }\end{array}$ & $\begin{array}{c}\text { Temperature } \\
\left({ }^{\circ} \mathrm{C}\right)\end{array}$ & Other Data \\
\hline 25 & 289 & 0.09 & 35 & \multirow{8}{*}{ 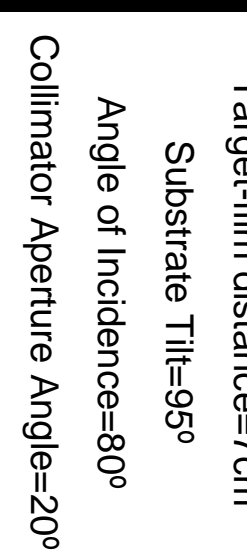 } \\
\hline 100 & 285 & 0.35 & 75 & \\
\hline 125 & 286 & 0.44 & 80 & \\
\hline 150 & 287 & 0.52 & 85 & \\
\hline 200 & 294 & 0.68 & 95 & \\
\hline 300 & 307 & 0.98 & 110 & \\
\hline 400 & 316 & 1.26 & 135 & \\
\hline 100 & 295 & 0.36 & 150 & \\
\hline
\end{tabular}


Figure 1
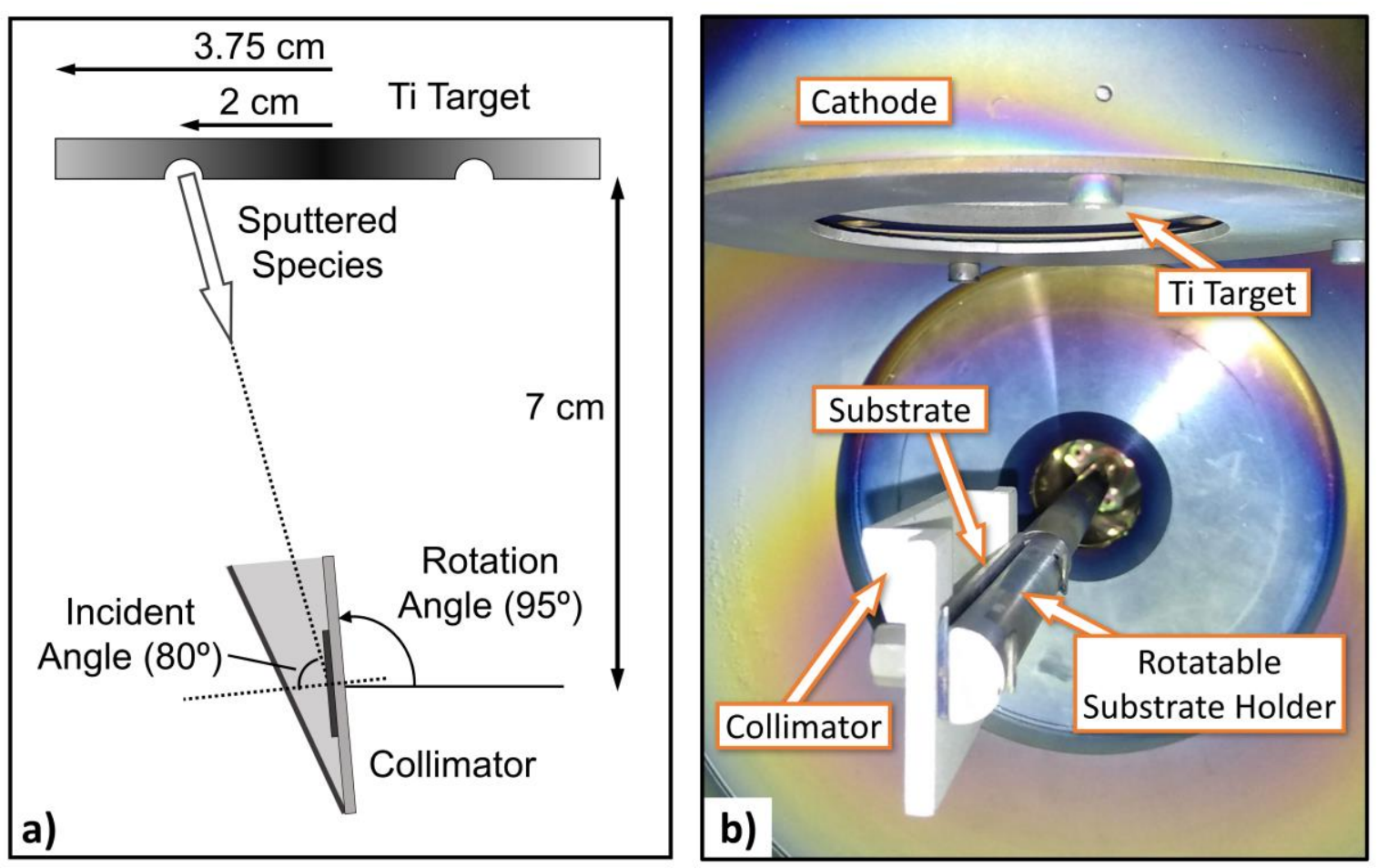
Figure 2
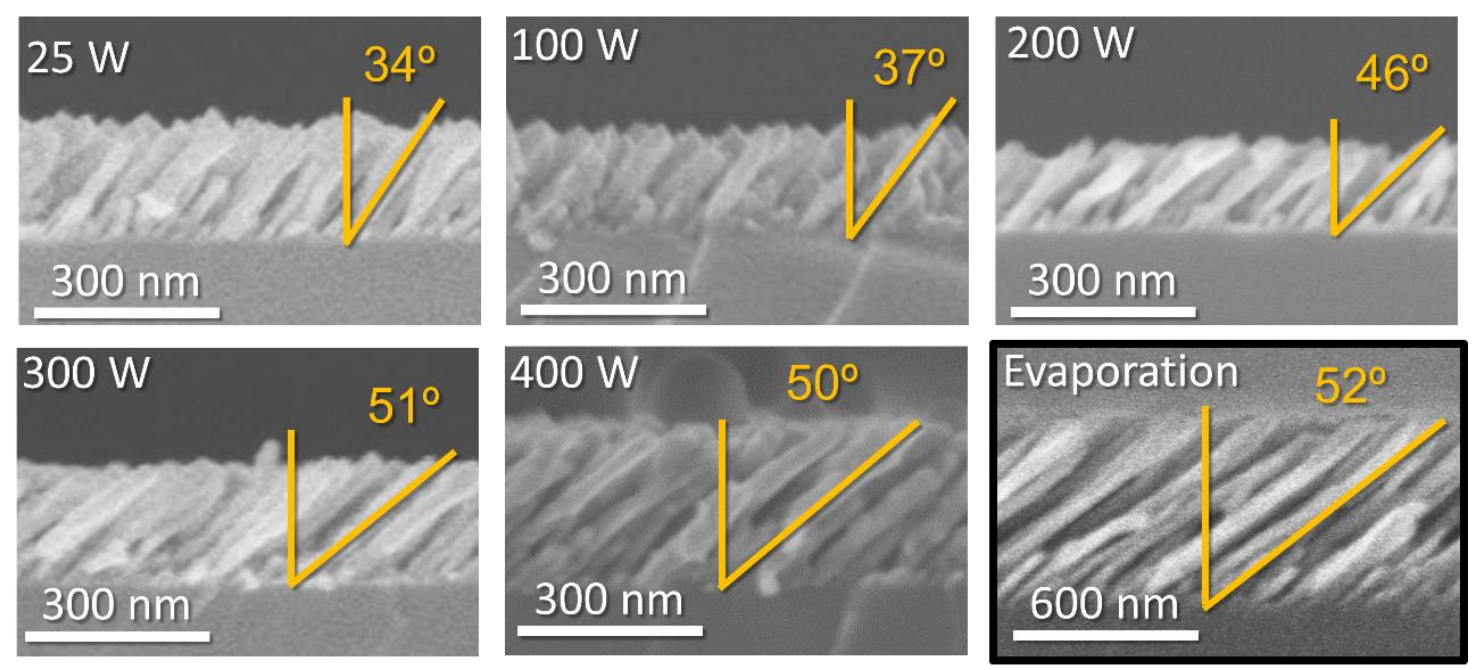
Figure 3
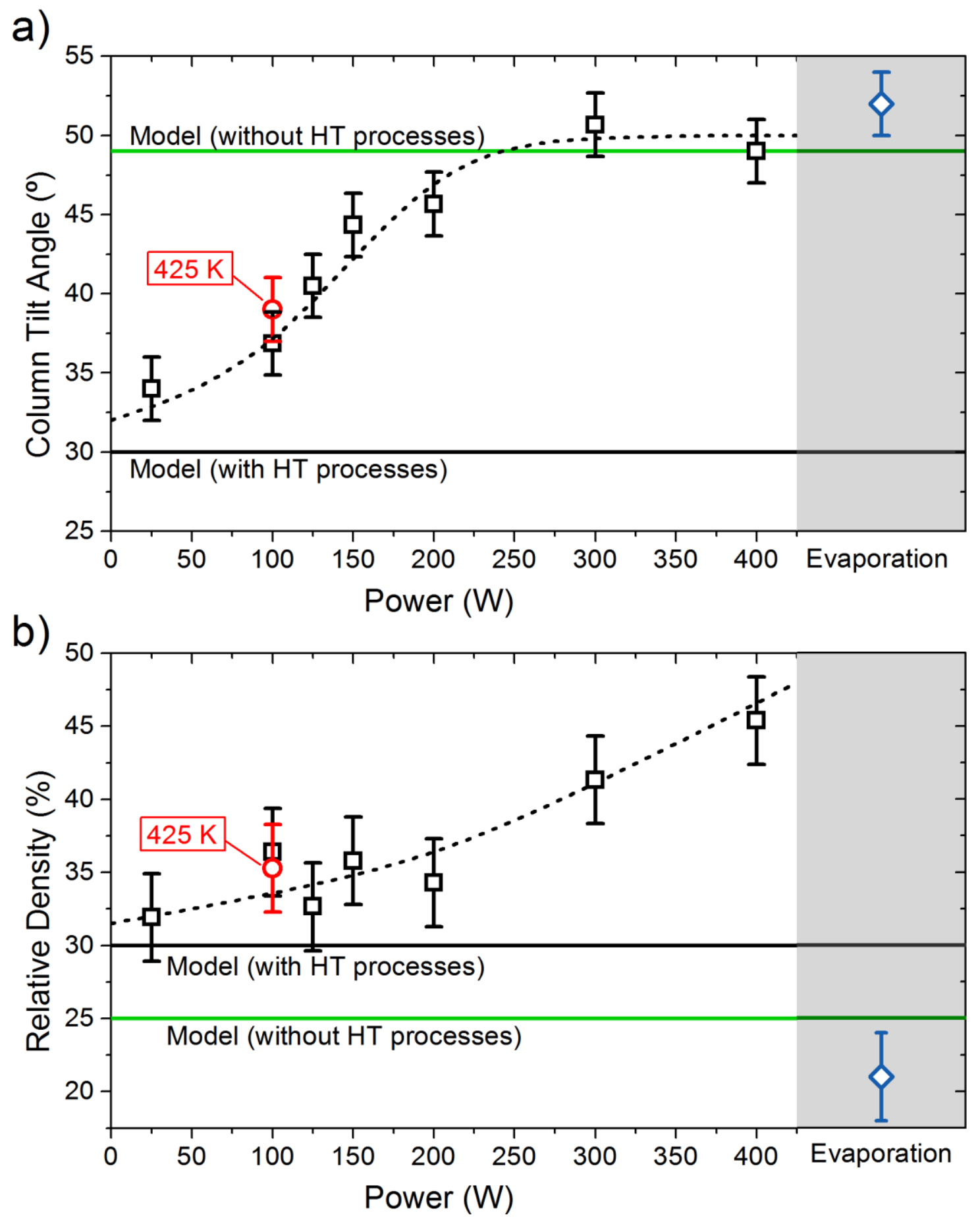
Figure 4
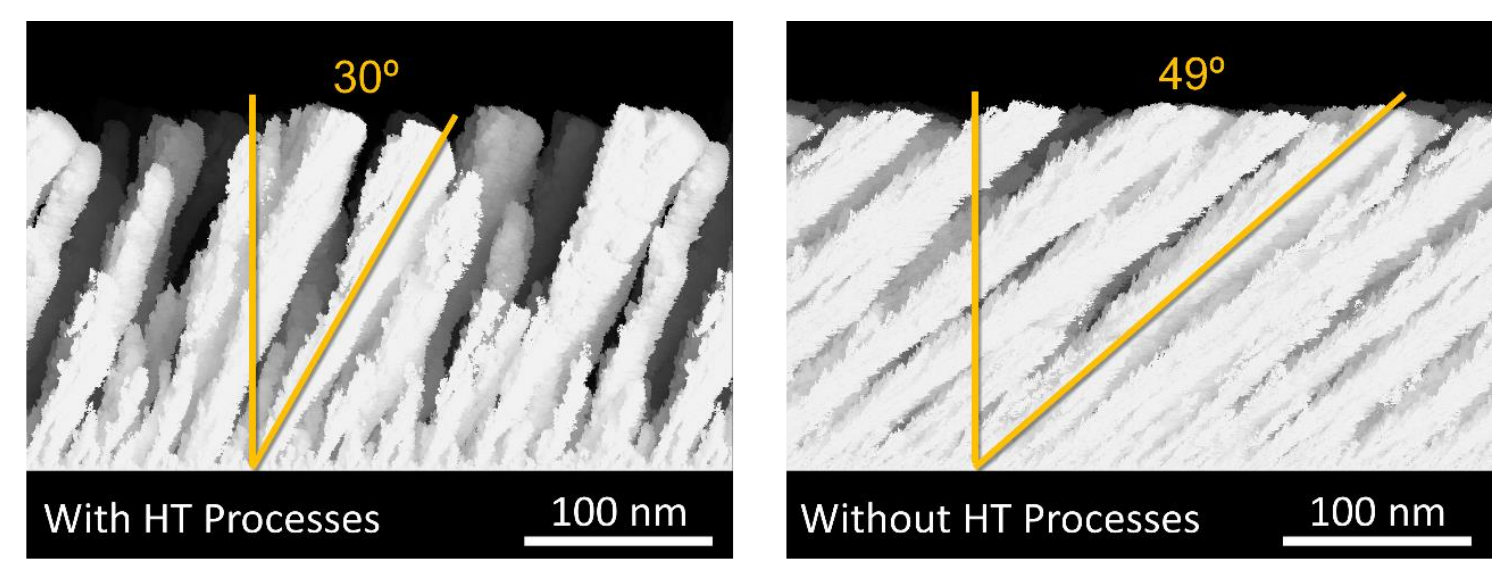
Figure 5

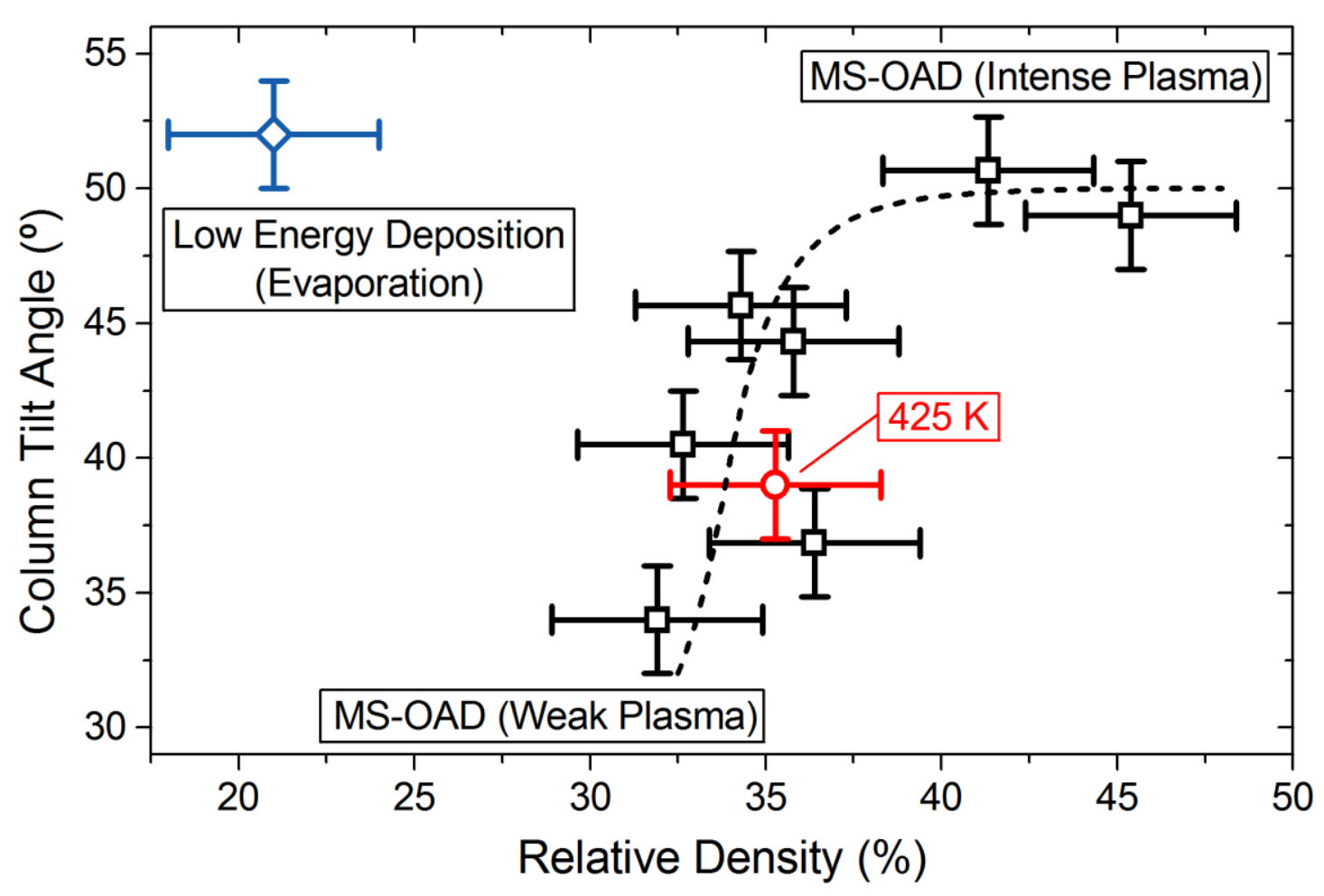


Figure 6

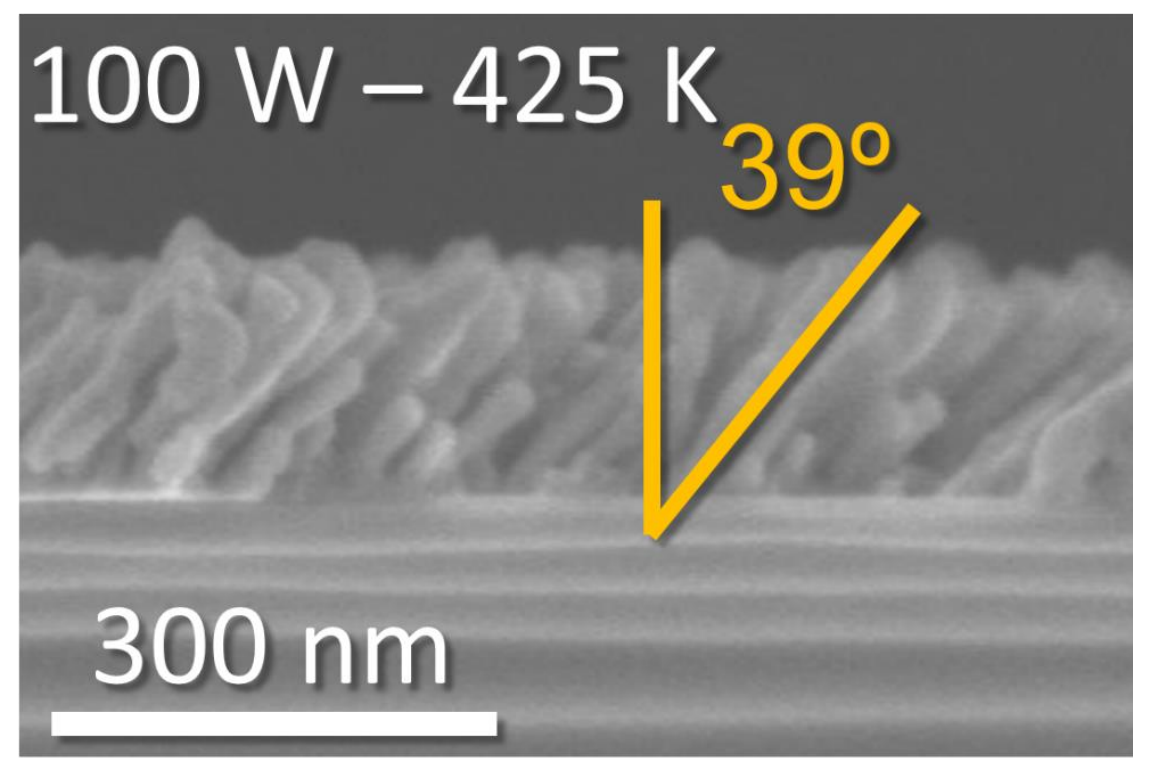


Figure 7

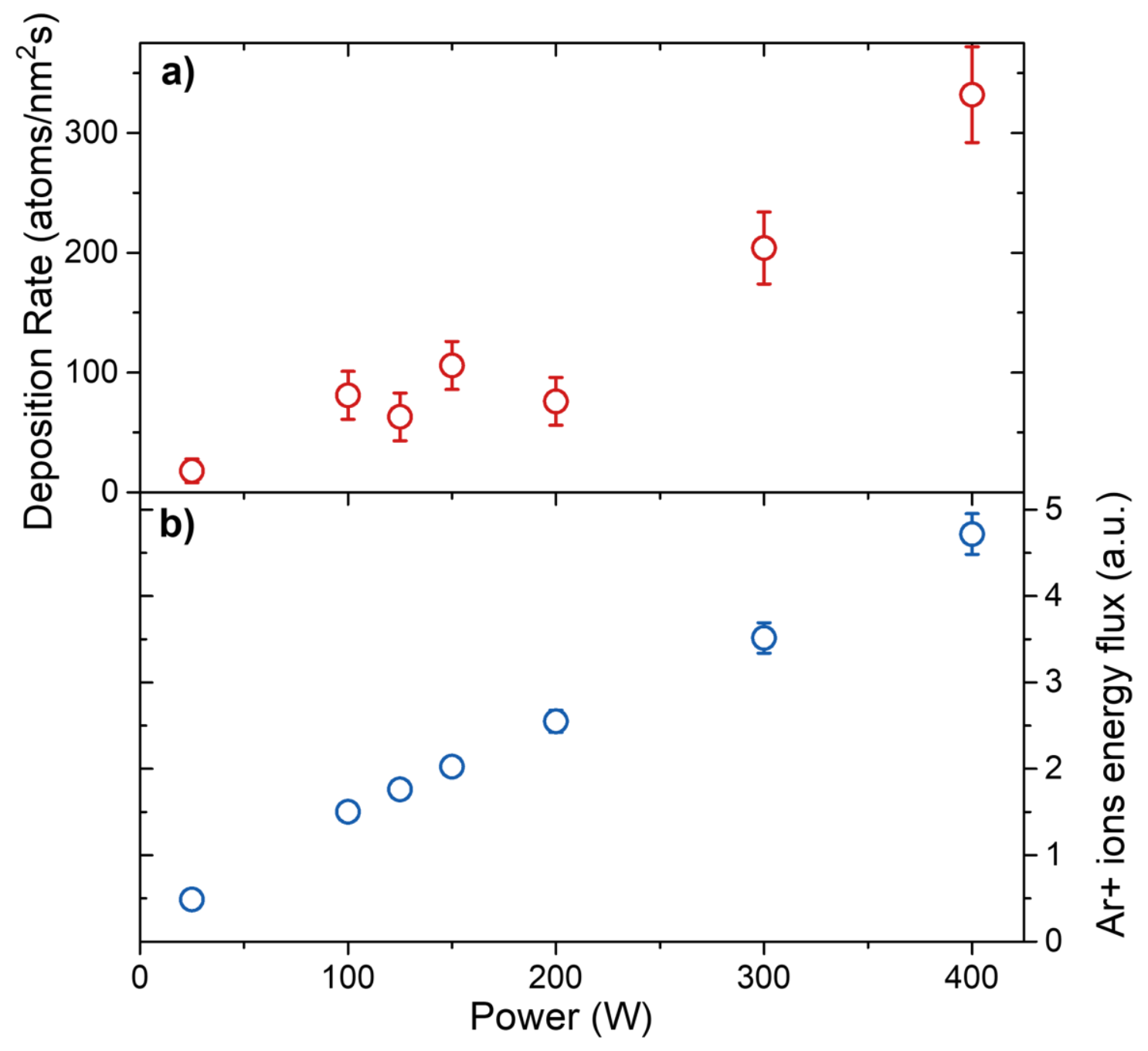


Figure 8

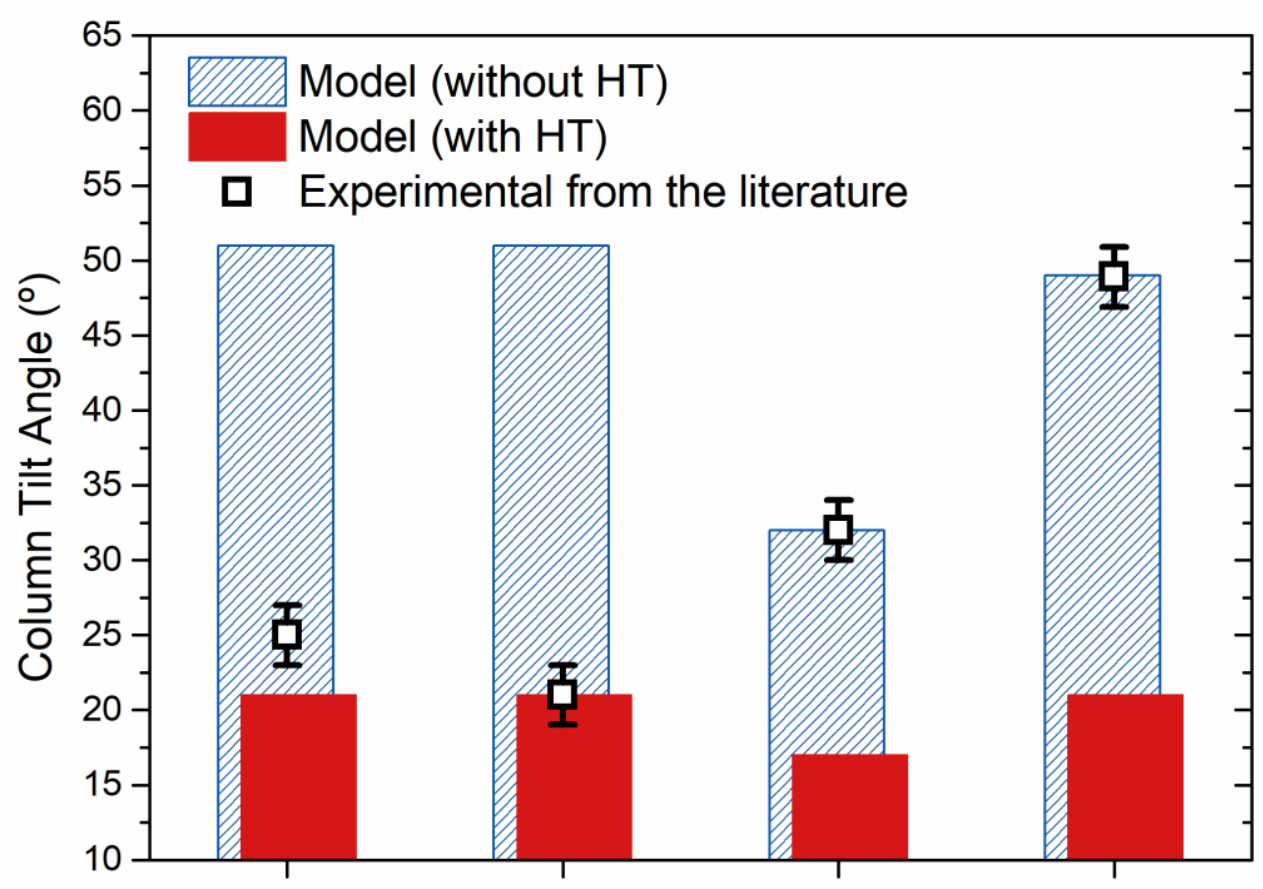

Alvarez et al. Medina-Cruz et al. Medina-Cruz et al. Dervaux et al. [27] [30] (System 1) [30] (System 2) [28] 